\title{
Cerebral blood flow characteristics in patients with post-lumbar puncture headache
}

\author{
Magdalena Nowaczewska • Barbara Książkiewicz
}

Received: 2 June 2011/Revised: 12 August 2011/Accepted: 30 August 2011/Published online: 21 September 2011

(C) The Author(s) 2011. This article is published with open access at Springerlink.com

\begin{abstract}
The aim of this study was to verify if diagnostic lumbar puncture (DLP) in post-lumbar puncture headache (PLPH) patients is related to significant changes in cerebral blood flow which could be visualized by transcranial Doppler (TCD). Sixty-six patients were enrolled in this study. TCD was performed $24 \mathrm{~h}$ before DLP and repeated within $24 \mathrm{~h}$ after the procedure. The measurements included mean velocity $\left(V_{\text {mean }}\right)$, peak systolic velocity $\left(V_{\max }\right)$, and Gosling's pulsatility index (PI), in the left and right middle cerebral artery (MCA). PLPH was observed in 21 patients $(32 \%)$. No significant differences were noted in $V_{\text {mean }}, V_{\max }$ and PI between the right and left MCAs-both before DLP and following this procedure. In patients who developed PLPH, bilateral pre-puncture values of $V_{\text {mean }}$ and $V_{\max }$ were significantly higher and PI was significantly lower compared to unaffected individuals. No significant differences were observed between these groups in terms of post-puncture $V_{\text {mean }}$ and $V_{\text {max }}$, but the post-puncture PI was still significantly lower in PLPH cases. In PLPH cases, the post-puncture values of $V_{\text {mean }}$ and $V_{\max }$ were significantly lower than the respective baseline parameters. A significant inverse correlation was present between PLPH severity and bilateral pre-puncture PI. In conclusion, this study revealed that higher baseline values of $V_{\text {mean }}$ and $V_{\max }$ and low PI in bilateral MCAs predispose patients to PLPH.
\end{abstract}

Keywords Cerebral hemodynamics - Flow velocity . Intracranial pressure · Post-lumbar puncture headache . Transcranial Doppler

M. Nowaczewska $(\bowtie) \cdot$ B. Książkiewicz Department of Neurology, Nicolaus Copernicus University in Torun, Ludwik Rydygier Collegium Medicum in Bydgoszcz, ul. M. Skłodowskiej-Curie 9, 85-094 Bydgoszcz, Poland e-mail: magy_mat@by.onet.pl

\section{Introduction}

Diagnostic lumbar puncture (DLP) is a frequent procedure used in the diagnosis of neural system disorders. Postlumbar puncture syndrome is a common complication of DLP occurring in about $3-40 \%$ of patients [1-4]. Postlumbar puncture headache (PLPH) is the predominant symptom of this syndrome, probably resulting from the leakage of cerebrospinal fluid into the epidural cavity through the post-puncture opening in the dura mater. The loss of cerebrospinal fluid causes a drop off in intracranial pressure (ICP), causing compensative dilatation of cerebral vessels, which is reflected by the headache $[1-3,5]$. This theory has one significant weakness, namely that PLPH do not occur in every patient following DLP.

Transcranial Doppler ultrasonography (TCD) enables us to assess blood flow in the intracranial arteries non-invasively. Assuming that TCD parameters are influenced both by changes in cerebral vessel diameters and by fluctuations in ICP [6-8], we aimed to verify if DLP resulted in significant changes in cerebral blood flow (CBF) that could be visualized by TCD. Therefore, we identified a group of subjects who developed PLPH following DLP and compared their pre- and post-puncture blood flow parameters with respective values of PLPH-free subjects.

\section{Methods}

Participants and exclusion criteria

This study included patients at the Department of Neurology, who required a scheduled DLP. Exclusion criteria from this group included emergency lumbar punctures, inadequate temporal bone windows, stenosis of the middle 
cerebral arteries, hemodynamically significant stenosis of the common carotid arteries, atrial fibrillation, and patients who were immobilized.

All the procedures were approved by the Local Ethics Committee of the Ludwik Rydygier Collegium Medicum in Bydgoszcz. The subjects gave their informed consent before the start of any procedure.

\section{Study procedures}

TCD was performed $24 \mathrm{~h}$ before DLP and repeated within $24 \mathrm{~h}$ after the procedure. Lumbar puncture was done following standard protocol by one of 11 physicians employed at the department. PLPH was diagnosed according to the International Classification of Headache Disorders (ICH-II) guidelines [9]. The presence and severity of PLPH was evaluated for 5 days, on a daily basis, using a numeric rating scale (NRS).

Sixty-six patients were enrolled to this study, including 37 men and 29 women. The mean age of these patients was $47.4 \pm 16.5$ years (range from 16 to 88, Table 1). Patients were subjected to diagnostic procedures due to cranial mononeuropathy $(n=12)$, polyneuropathy $(n=7)$, multiple sclerosis $(n=13)$, vertigo $(n=7)$, diffuse injuries of the brain or spinal cord $(n=9)$, epilepsy $(n=5)$, and other reasons.

Lumbar puncture was performed in the lateral recumbent position, using the intervertebral space between L4 and L5 or between L5 and S1, with the aid of a 22-gauge needle (BD Spinal Needle Quincke Type Point). The volumes of collected cerebral fluid ranged from around 6-10 ml. All the procedures were performed between 11 and 12 a.m. Following the procedure, the patients were ordered to stay in bed for $8 \mathrm{~h}$.

TCD was performed using a Pioneer apparatus (Nicolet/ EME) with a $2-\mathrm{MHz}$ probe. Patients were examined in a supine position. Following localization of the temporal window, the middle cerebral artery (MCA) was identified. The probe was placed so as to record the peak possible velocity from the vessel. The measurements were repeated three times with the probe fixed in this position. The measurements included mean velocity $\left(V_{\text {mean }}\right)$, peak systolic velocity $\left(V_{\max }\right)$, and Gosling's pulsatility index $(\mathrm{PI})$ at 54-56 mm, in the left and right MCA. All TCDs were performed by the same physician being experienced in neurosonology and unaware of PLPH presence and severity in patients examined.

\section{Statistics}

Continuous variables were presented as arithmetic means and their standard deviations. Mean values of normally distributed variables were compared with Student's $t$ test for independent samples, whereas the variables whose distributions were not normal were compared with the nonparametric Mann-Whitney $U$ test. Dependent variables were compared with the Student's $t$ test for dependent samples. Distributions of discrete variables were compared between the groups with the aid of $\chi^{2}$ test. Associations between pairs of variables were tested with Spearman's coefficient of correlation. Calculations were performed using Statistica 7 (StatSoft ${ }^{\circledR}$, Poland) software, and statistical significance was defined as $p \leq 0.05$.

\section{Results}

Post-lumbar puncture headache was observed in 21 out of 66 patients who underwent DLP (32\%), whereas symptoms of post-lumbar puncture syndrome were not noted in the remaining 45 subjects $(68 \%)$. In most patients $(86 \%)$, the symptoms manifested on the first day following DLP,
Table 1 Clinical characteristics of patients who developed postlumbar puncture headache $(\mathrm{PLPH})$ and in PLPH-free individuals

\begin{tabular}{llll}
\hline Parameter & PLPH-free $(n=45)$ & PLPH $(n=21)$ & $p$ value \\
\hline Age in years, mean \pm SD & $49.3 \pm 17.8$ & $43.2 \pm 12.5$ & 0.16 \\
Women, $n(\%)$ & $13(28.9)$ & $16(76.2)$ & 0.00031 \\
Men, $n(\%)$ & $32(71.1)$ & $5(23.8)$ & 0.00026 \\
History of headache, $n(\%)$ & $9(20)$ & $14(66.7)$ & 0.00021 \\
Tension-type headache, $n(\%)$ & $6(13.3)$ & $11(52)$ & 0.00034 \\
Migraine without aura, $n(\%)$ & $3(6.7)$ & $2(9.5)$ & 0.21 \\
Other type of headache, $n(\%)$ & $0(0)$ & $1(4.8)$ & - \\
Arterial hypertension, $n(\%)$ & $12(26.67)$ & $3(14.29)$ & 0.26 \\
Diabetes, $n(\%)$ & $9(20)$ & $2(9.5)$ & 0.29 \\
Hypercholesterolemia, $n(\%)$ & $15(33.3)$ & $4(19.1)$ & 0.23 \\
Hematocrit in $\%$, mean \pm SD & $41.8 \pm 3.3$ & $39.1 \pm 3.9$ & 0.006 \\
Hemoglobin in g/l, mean \pm SD & $14.2 \pm 1.3$ & $13.0 \pm 1.5$ & 0.001 \\
\hline
\end{tabular}


Table 2 Pre- and post-puncture values (mean $\pm \mathrm{SD}$ ) of mean velocity $\left(V_{\text {mean }}\right)$, peak systolic velocity $\left(V_{\max }\right)$, and Gosling's pulsatility index (PI) in the right (MCA R) and left (MCA L) middle cerebral arteries of patients who developed post-lumbar puncture headache (PLPH) and in PLPH-free individuals

\begin{tabular}{|c|c|c|c|}
\hline & PLPH-free $(n=45)$ & PLPH $(n=21)$ & $p$ value \\
\hline Pre-puncture $V_{\text {mean }}$, MCA R $(\mathrm{m} / \mathrm{s})$ & $55.2 \pm 9.7$ & $71.9 \pm 24.9$ & 0.0002 \\
\hline Post-puncture $V_{\text {mean }}$, MCA R $(\mathrm{m} / \mathrm{s})$ & $55.5 \pm 12.6$ & $58.4 \pm 20.2$ & 0.47 \\
\hline$p$ value & 0.86 & 0.0004 & - \\
\hline Pre-puncture $V_{\text {mean }}$, MCA L $(\mathrm{m} / \mathrm{s})$ & $55 \pm 12.01$ & $69.12 \pm 22.56$ & 0.0015 \\
\hline Post-puncture $V_{\text {mean }}$, MCA L (m/s) & $54.2 \pm 13.2$ & $59.4 \pm 19.1$ & 0.19 \\
\hline$p$ value & 0.70 & 0.008 & - \\
\hline Pre-puncture $V_{\max }$, MCA R (m/s) & $89.9 \pm 16.2$ & $109.3 \pm 36.9$ & 0.004 \\
\hline Post-puncture $V_{\max }$, MCA R $(\mathrm{m} / \mathrm{s})$ & $91.1 \pm 21.5$ & $89.8 \pm 30.1$ & 0.84 \\
\hline$p$ value & 0.70 & 0.0002 & - \\
\hline Pre-puncture $V_{\max }$, MCA L $(\mathrm{m} / \mathrm{s})$ & $89.8 \pm 19.4$ & $104.3 \pm 32.2$ & 0.03 \\
\hline Post-puncture $V_{\max }$, MCA L $(\mathrm{m} / \mathrm{s})$ & $88.7 \pm 20.4$ & $90.7 \pm 28.0$ & 0.74 \\
\hline$p$ value & 0.74 & 0.009 & - \\
\hline Pre-puncture PI, MCA R & $0.99 \pm 0.20$ & $0.86 \pm 0.14$ & 0.01 \\
\hline Post-puncture PI, MCA R & $1.0 \pm 0.21$ & $0.89 \pm 0.12$ & 0.03 \\
\hline$p$ value & 0.46 & 0.41 & - \\
\hline Pre-puncture PI, MCA L & $0.98 \pm 0.22$ & $0.84 \pm 0.13$ & 0.01 \\
\hline Post-puncture PI, MCA L & $0.99 \pm 0.23$ & $0.86 \pm 0.13$ & 0.01 \\
\hline$p$ value & 0.43 & 0.65 & - \\
\hline
\end{tabular}

on average $17.7 \pm 10.2 \mathrm{~h}$ after the procedure (range $7-44 \mathrm{~h}$ ). The mean pain score was 5.1 on NRS. PLPH persisted $5.5 \pm 1.8$ days on average (range 2-10 days). Female patients predominated amongst the PLPH cases (16, compared to five men, $p<0.0003$ ). Most patients who developed PLPH $(n=14)$ had a history of headaches, mostly tension headaches. The mean age of patients with PLPH was lower than for unaffected individuals (43.2 vs. 49.3 years), but this difference was insignificant (Table 1).

In both groups of patients, no significant differences were observed in $V_{\text {mean }}, V_{\max }$ and PI between the right and left MCAs_-both before DLP and following this procedure (Table 2).

In patients who developed PLPH, bilateral pre-puncture values of $V_{\text {mean }}$ and $V_{\text {max }}$ were significantly higher and PI was significantly lower compared to unaffected individuals. No significant differences were observed between these groups in terms of post-puncture $V_{\text {mean }}$ and $V_{\text {max }}$, but the post-puncture PI was still significantly lower in PLPH cases (Table 2). Moreover, hemoglobin concentration and hematocrit values were significantly lower in patients who developed headache compared to unaffected individuals (Table 1).

In PLPH cases, the post-puncture values of $V_{\text {mean }}$ and $V_{\max }$ were significantly lower than the respective baseline parameters. Significant puncture-related changes in bilateral $V_{\text {mean }}$ and $V_{\max }$ were not observed in patients who did not develop PLPH. After the puncture, a slight increase in
PI was observed bilaterally in both groups, but these changes lacked statistical significance (Table 2). When study results were stratified according to patient gender, significant post-puncture changes in $V_{\text {mean }}$ and $V_{\max }$ were observed only in women $(p<0.01$ and $p<0.07$ in right and left MCA, respectively).

A significant inverse correlation was present between PLPH severity on NRS and bilateral pre-puncture PI (Fig. 1).

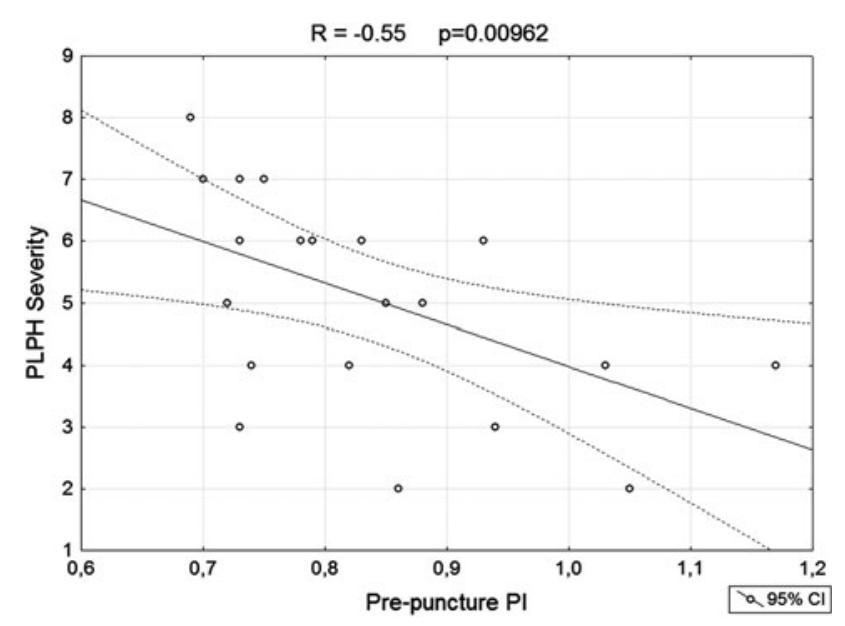

Fig. 1 Correlation between post-lumbar puncture headache (PLPH) severity on numeric rating scale and pre-puncture Gosling's pulsatility index (PI) in the left middle cerebral artery 


\section{Discussion}

Post-lumbar puncture headache is believed to result from cerebrospinal fluid leakage through the post-puncture opening, with a subsequent decrease in intracranial pressure and cerebral vessel dilatation $[1-3,5]$. This theory was confirmed by our study. Following the puncture, we observed significant decreases in $V_{\text {mean }}$ and $V_{\max }$ in bilateral MCAs of patients who developed PLPH when compared to their respective pre-puncture values. The drop off in $V_{\text {mean }}$ and $V_{\max }$ observed in this group of patients may result from the dilatation of intracranial vessels, a consequence of lowered ICP. Similar puncture-related decreases in $V_{\max }$ in patients with PLPH were previously described by Gobel et al. [10], but only affecting the right MCA.

In our study, significant puncture-related changes in $V_{\text {mean }}$ and $V_{\max }$ were not observed in bilateral MCAs of PLPH-free individuals. Presumably, the loss of cerebrospinal fluid in these patients was not significant enough to be reflected by compensative dilatation of cerebral vessels.

Pulsatility index is an indirect indicator of the ICP changes. The value of PI illustrates the resistance of a vascular bed (supplied by a given artery) when examined by TCD. There is a linear relationship between changes in PI and ICP. A decrease in PI may result both from lowered ICP and from vascular dilatation [7, 8]. Assuming that the loss of cerebrospinal fluid with further decrease in ICP is the reason for PLPH, PI should decrease during the early phase of post-lumbar puncture syndrome. Reactive dilatation of cerebral vessels due to decreased ICP should result in further PI drop off. However, this theory was not confirmed by our study. We did not observe significant puncture-related changes in PI, both in patients who developed PLPH and in unaffected individuals. Presumably, ICP fluctuations were slight or transient and therefore we failed to record them.

Noticeably, baseline (pre-puncture) PI values in patients who later developed PLPH were significantly lower compared to subjects free of this condition. This finding suggests that compensative dilatation of cerebral vessels does not accompany every decrease in ICP but is rather characterized by low values of this parameter. There exist linear relationships between PI or $V_{\max }$ changes and changes in ICP in cerebral vessels. PI is known to decrease and $V_{\max }$ to increase with lowered ICP [7, 8]. Thus, it is likely that the baseline ICP in patients who later developed PLPH was lower than in unaffected individuals. Consequently, further ICP drop off due to cerebrospinal fluid loss might result in compensative vascular dilatation in patients with PLPH. A relatively higher baseline ICP in PLPH-free individuals might in turn protect them against intracranial 'hypotension' and resulting post-puncture headache. This theory was supported by a significant correlation observed in our study, between pre-puncture PI and PLPH severity: the lower the baseline PI (and indirectly-ICP) the more severe the PLPH.

In cases with PLPH, baseline $V_{\text {mean }}$ and $V_{\max }$ values were higher and PI lower than in unaffected individuals. What factors, beside different ICPs, could be responsible for these differences? $V_{\text {mean }}$ and $V_{\text {max }}$ are known to decrease with age $[6,11]$. Consequently, higher baseline values of these parameters in PLPH patients might be associated with the higher age of this group, compared to unaffected individuals. In our study, age differences between these two groups were insignificant, and therefore, unlikely to be responsible for the results found in this experiment.

The predominance of women amongst the PLPH patients might be another potential reason for flow velocity differences observed between affected and unaffected individuals. Higher $V_{\text {mean }}$ and $V_{\max }$ values were previously demonstrated in females compared to males and these differences were attributed to higher values of CBF in women [11, 12].

Additionally, our study revealed significantly lower hematocrit and hemoglobin values in PLPH subjects than in unaffected individuals. A lowered hematocrit is known to enhance flow velocity [13], and decreases PI due to reduced resistance. Consequently, decreased hematocrit values might be responsible for higher values of $V_{\text {mean }}$ and $V_{\max }$ and lower PI observed in the PLPH cases. However, according to Adams et al. [14], hematocrit values do not influence flow velocities unless they are below $35 \%$.

In conclusion, the findings of this study partially confirm the theory behind the etiology of PLPH stated in the introduction, and suggest that higher baseline values of $V_{\text {mean }}$ and $V_{\max }$ and low PI in bilateral MCAs predispose patients to this condition. Moreover, the severity of headache was confirmed to correlate with PI values. Additional risk factors of PLPH were observed to include female gender, history of headaches, and decreased hematocrit and hemoglobin levels. Finally, within the $24 \mathrm{~h}$ following puncture, significant decreases in $V_{\text {mean }}$ and $V_{\max }$ were observed in bilateral MCAs of patients who developed PLPH.

Conflict of interest The authors declare that they have no conflicts of interest.

Open Access This article is distributed under the terms of the Creative Commons Attribution Noncommercial License which permits any noncommercial use, distribution, and reproduction in any medium, provided the original author(s) and source are credited.

\section{References}

1. Spielman FJ (1982) Post-lumbar puncture headache. Headache 22:280-283

2. Silberstein SD (1998) Post-lumbar puncture headache. Cephalalgia $18: 72$ 
3. Bezov D, Lipton RB, Ashina S (2010) Post-dural puncture headache: part I diagnosis, epidemiology, etiology, and pathophysiology. Headache 50:1144-1152

4. Zetterberg H, Tullhög K, Hansson O, Minthon L, Londos E, Blennow K (2010) Low incidence of post-lumbar puncture headache in 1,089 consecutive memory clinic patients. Eur Neurol 63:326-330

5. Hannerz J, Ericson K, Bro Skejo HP (1999) MR imaging with gadolinum in patients with and without post-lumbar puncture headache. Acta Radiol 40:135-141

6. Ackerstaff RG, Keunen RW, van Pelt W, Montauban van Swijndregt AD, Stijnen T (1990) Influence of biological factors on changes in mean cerebral blood flow velocity in normal ageing: a transcranial Doppler study. Neurol Res 12:187-191

7. Bellner J, Romner B, Reinstrup P, Kristiansson K-A, Ryding E, Brandt L (2004) Transcranial Doppler sonography pulsatility index (PI) reflects intracranial pressure (ICP). Surg Neurol 62:45-51

8. Voulgaris SG, Partheni M, Kaliora H, Haftouras N, Pessach IS, Polyzoidis KS (2005) Early cerebral monitoring using the transcranial Doppler pulsatility index in patients with severe brain trauma. Med Sci Monit 11:CR49-CR52

9. International Headache Society (2004) The international classification of headache disorders, 2nd edition. Cephalalgia 24(suppl 1): $1-160$
10. Göbel H, Klostermann H, Lindner V, Schenkl S (1990) Changes in cerebral haemodynamics in cases of post-lumbar puncture headache: a prospective transcranial Doppler ultrasound study. Cephalalgia 10:117-122

11. Müller M, Schimrigk K (1994) A comparative assessment of cerebral haemodynamics in the basilar artery and carotid territory by transcranial Doppler sonography in normal subjects. Ultrasound Med Biol 20:677-687

12. Oláh L, Valikovics A, Bereczki D, Fülesdi B, Munkácsy C, Csiba L (2000) Gender-related differences in acetazolamide-induced cerebral vasodilatory response: a transcranial Doppler study. J Neuroimaging 10:151-156

13. Isikay CT, Uzuner N, Gücüyener D, Ozdemir G (2005) The effects of hematocrit and age on transcranial Doppler measurements in patients with recent ischemic stroke. Neurol India 53:51-54

14. Adams RJ, McKie VC, Hsu L, Files B, Vichinsky E, Pegelow C, Abboud M, Gallagher D, Kutlar A, Nichols FT, Bonds DR, Brambilla D (1998) Prevention of a first stroke by transfusions in children with sickle cell anemia and abnormal results of transcranial Doppler ultrasonography. N Engl J Med 339:5-11 